\title{
Ordered mesoporous carbon confined highly dispersed PtCo alloy for oxygen reduction reaction: the effect of structure and composition on performance
}

Yifei Liao ${ }^{1,3}$, Yao Wang 2,3*, Jinchao Liu' ${ }^{1,3}$, Yiyun Tangl, Chaoling $W^{1,3}$, Yungui Chen $^{2,3 *}$

${ }^{1}$ Department of Advanced Energy Materials, College of Materials Science and Engineering, Sichuan University, Chengdu 610065, PR China.

${ }^{2}$ Institute of New Energy and Low-Carbon Technology, Sichuan University, Chengdu 610065, PR China.

${ }^{3}$ Engineering Research Center of Alternative Energy Materials \& Devices, Ministry of Education, Chengdu, 610065, PR China

* Corresponding authors: Yao Wang: wangyao516@scu.edu.cn; Yungui Chen, chenyungui@scu.edu.cn 

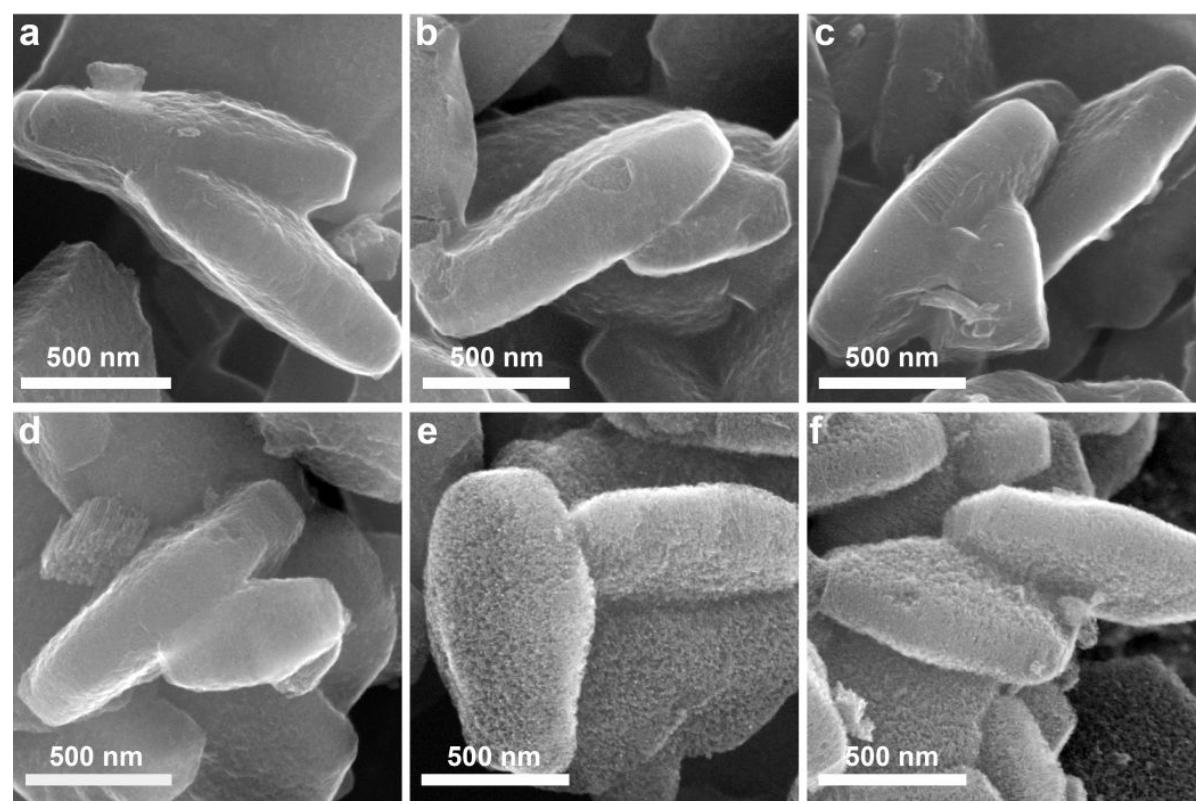

Figure S1. SEM images of a) PtCo@sOMC-300-1/2, b) PtCo@sOMC-500-1/2, c) PtCo@sOMC-700-1/2, d) PtCo@sOMC-800-1/2, e) PtCo@sOMC-900-1/2 and f) PtCo@sOMC-1100-1/2 catalysts.

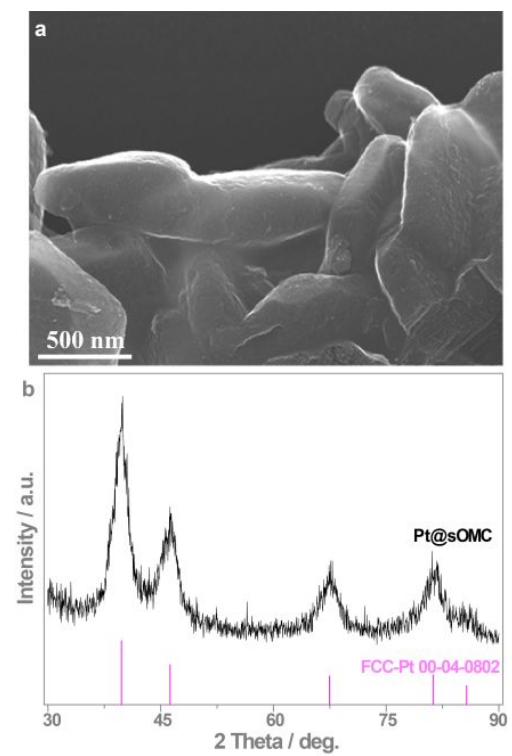

Figure S2. a) SEM image and b) XRD pattern of individual Pt@sOMC catalyst. 

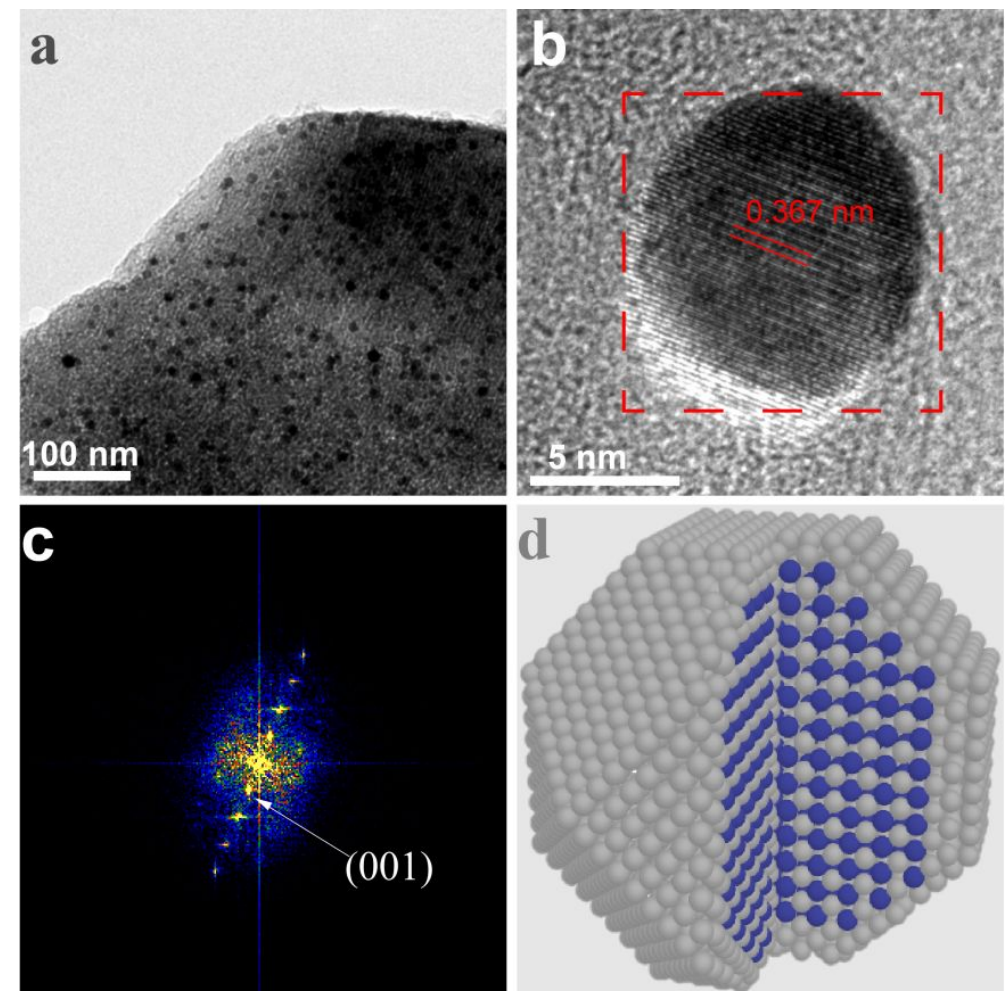

Figure S3. a) TEM image, b) HRTEM, and c) FFT patterns of PtCo@sOMC-11001/2 catalyst.
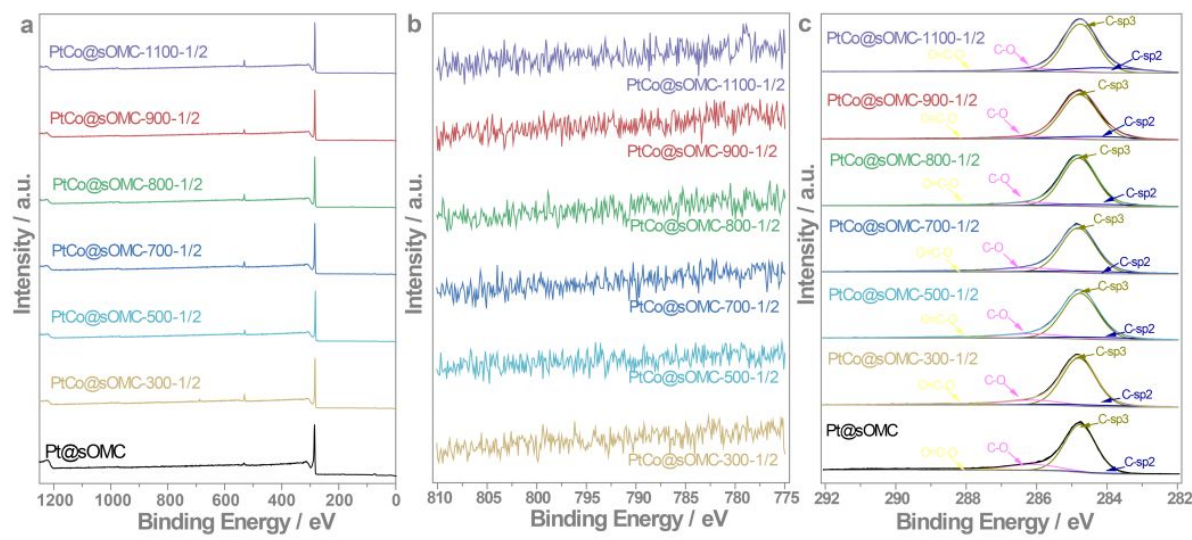

Figure S4. a) The survey spectra, b) decompressed Co $2 p$ spectra and c) decompressed C 1s spectra of PtCo@sOMC-T-1/2 catalysts.
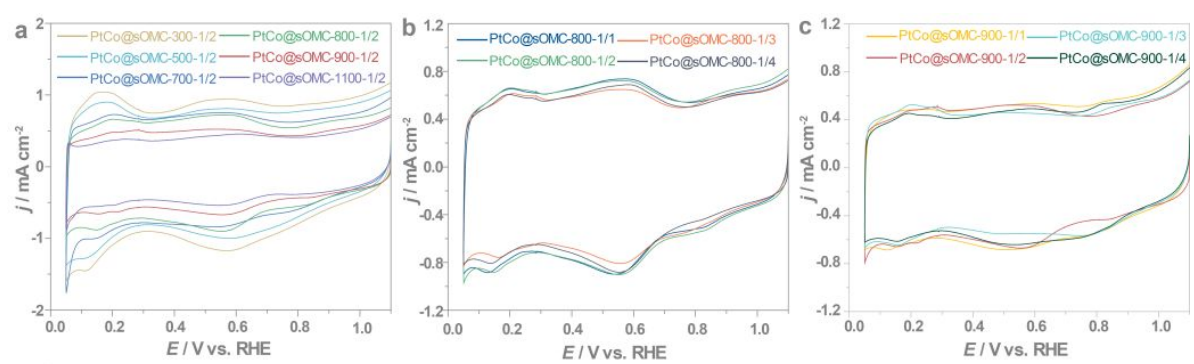

Figure S5. CV curves of PtCo@sOMC-T-1/x catalysts. 

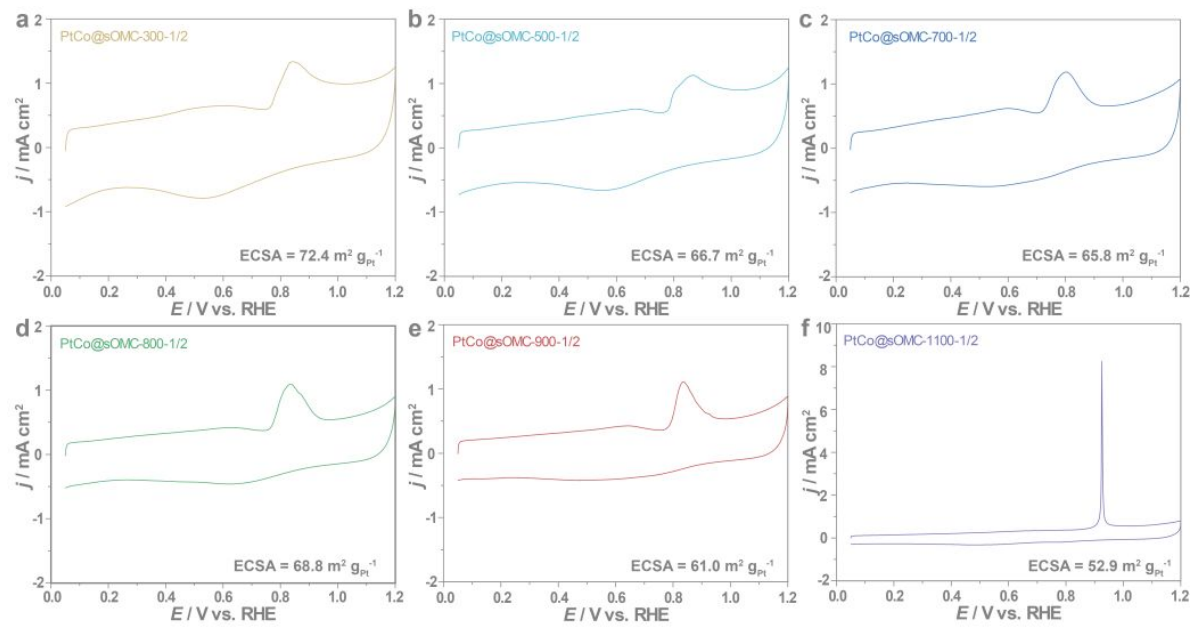

Figure S6. CO stripping curves of PtCo@sOMC-T-1/2 catalysts.
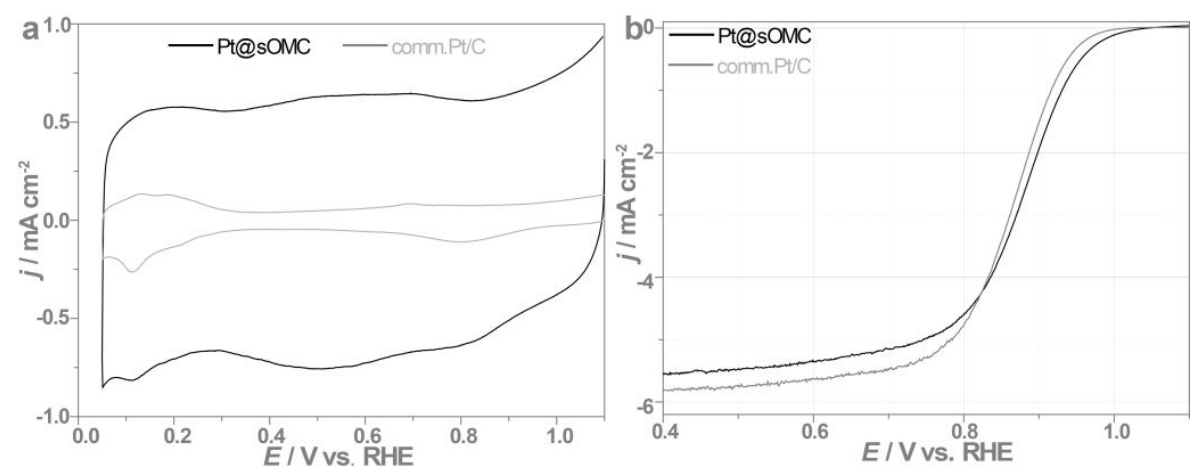

Figure S7. a) CV and b) LSV curves of Comm. Pt/C and Pt@sOMC catalysts.

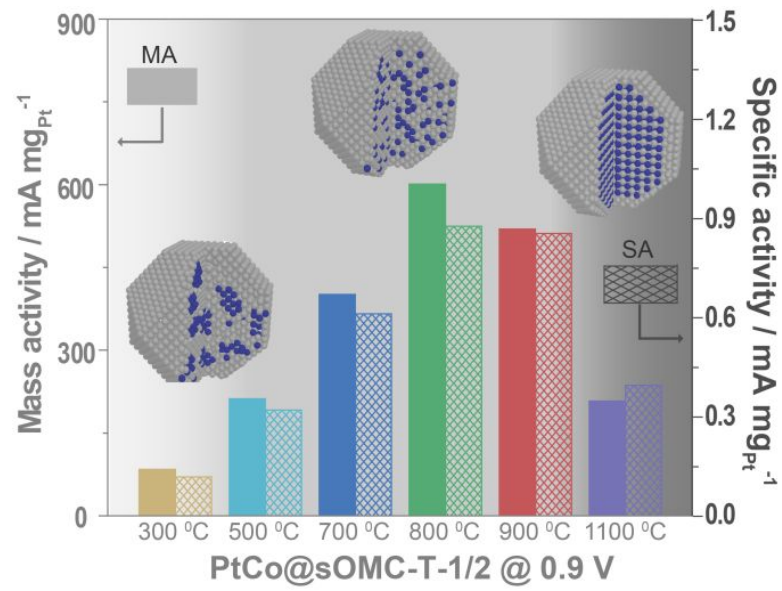

Figure S8. MA and SA at $0.9 \mathrm{~V}$ of $\mathrm{PtCo} @$ sOMC-T-1/2 catalysts. 

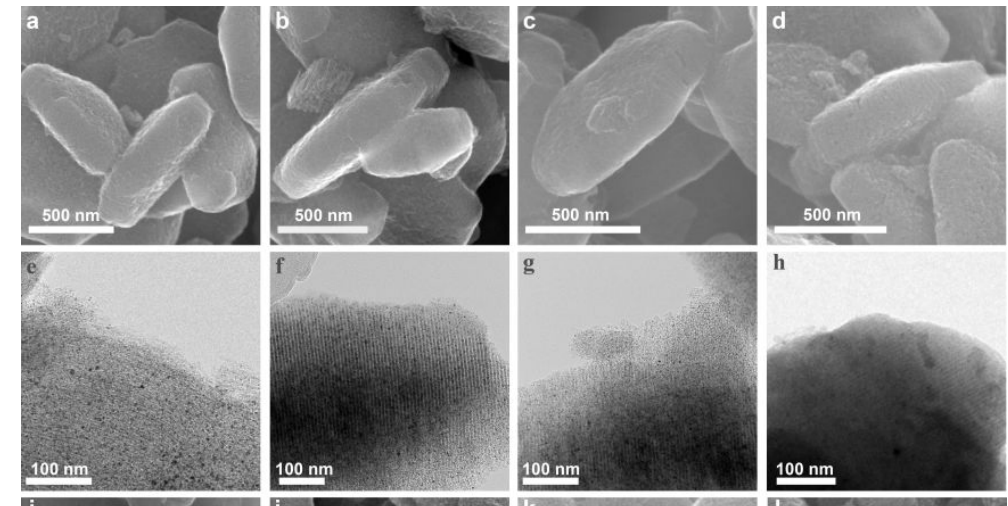

h
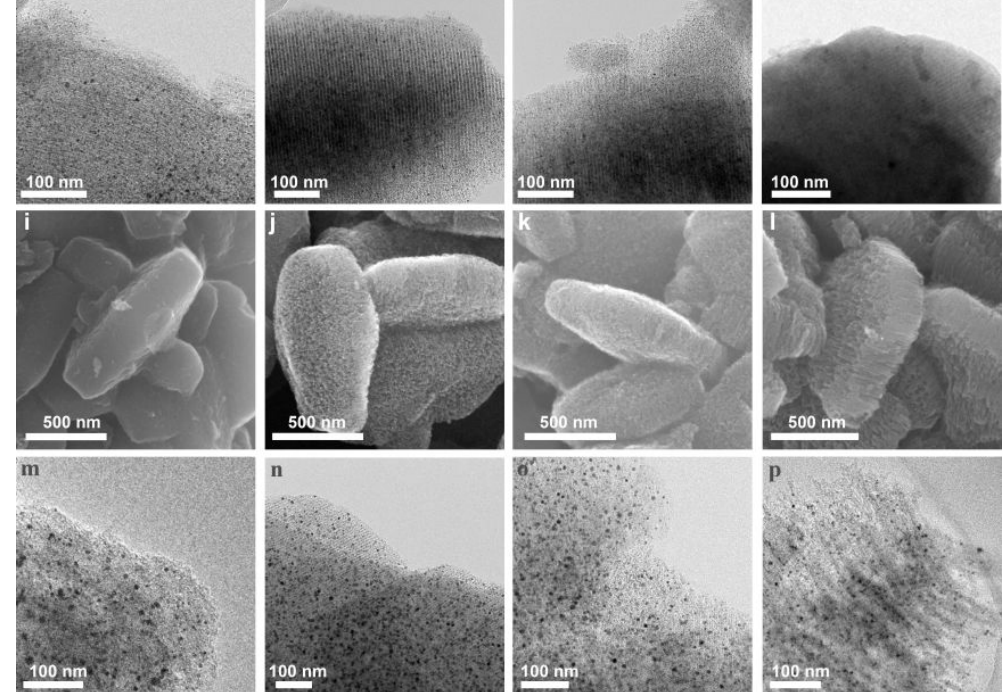

Figure S9. SEM and TEM images of a, e) PtCo@sOMC-800-1/1, b, f) PtCo@sOMC800-1/2, c, g) PtCo@sOMC-800-1/3, d, h) PtCo@sOMC-800-1/4, i, m)

PtCo@sOMC-900-1/1,j, n) PtCo@sOMC-900-1/2, k, p) PtCo@sOMC-900-1/3 and 1, p) PtCo@sOMC-900-1/4 catalysts.
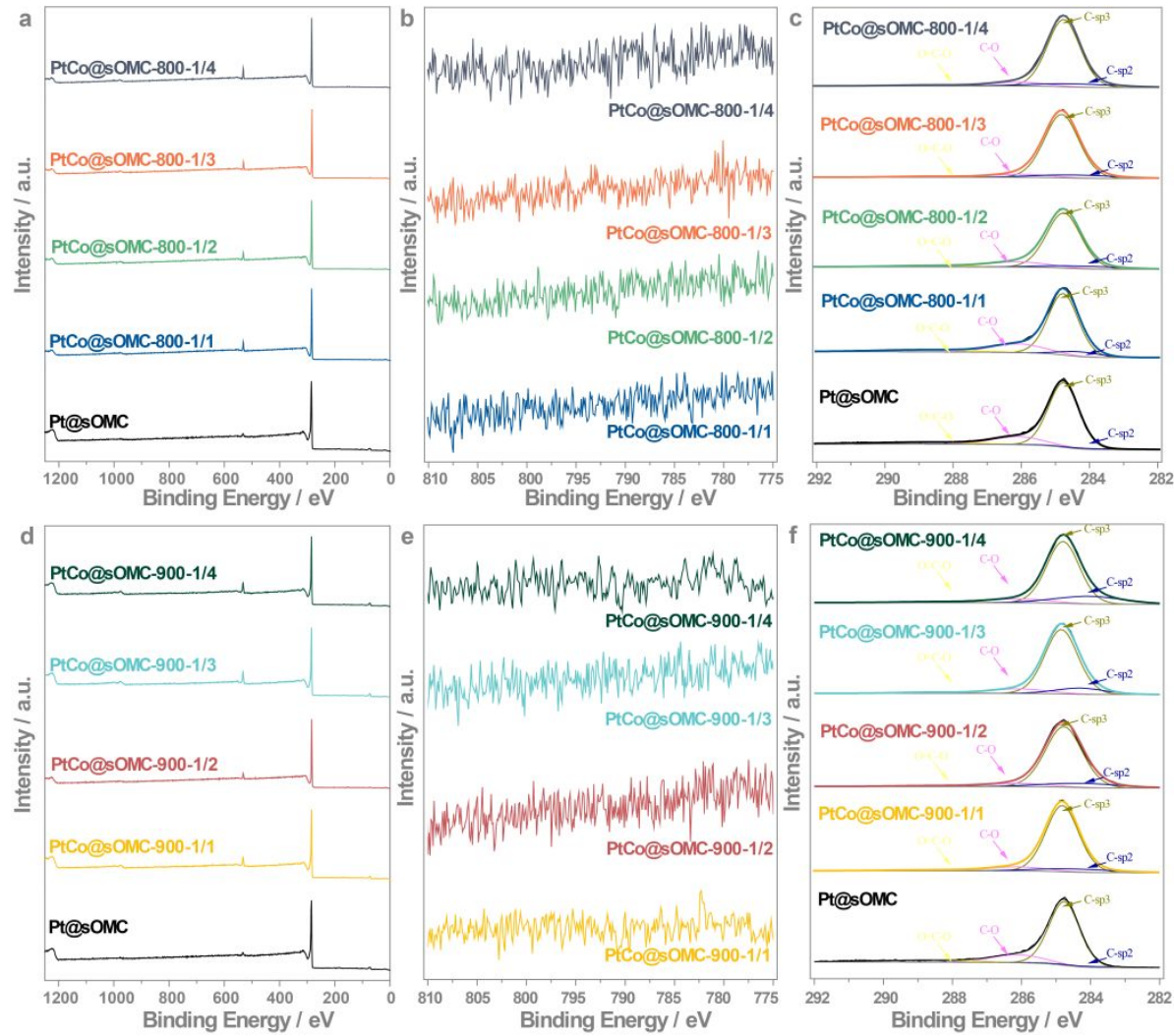
Figure S10. a) The survey spectra, b) decompressed Co $2 p$ spectra and c) decompressed C 1s spectra of PtCo@sOMC-800-1/x and PtCo@sOMC-900-1/x catalysts.

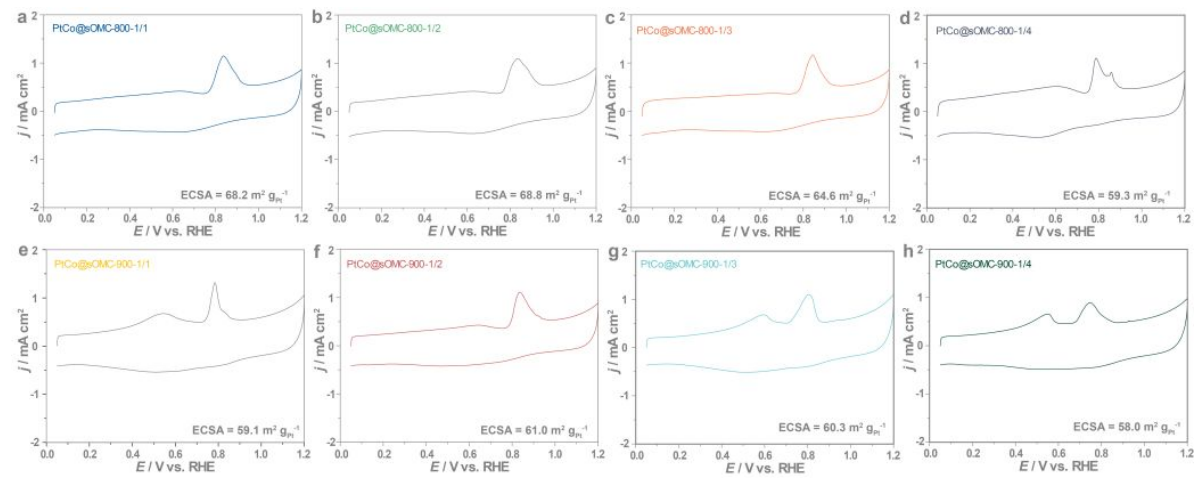

Figure S11. CO stripping curves of (a-d) PtCo@sOMC-800-1/x and (e-h)

PtCo@sOMC-900-1/x catalysts.
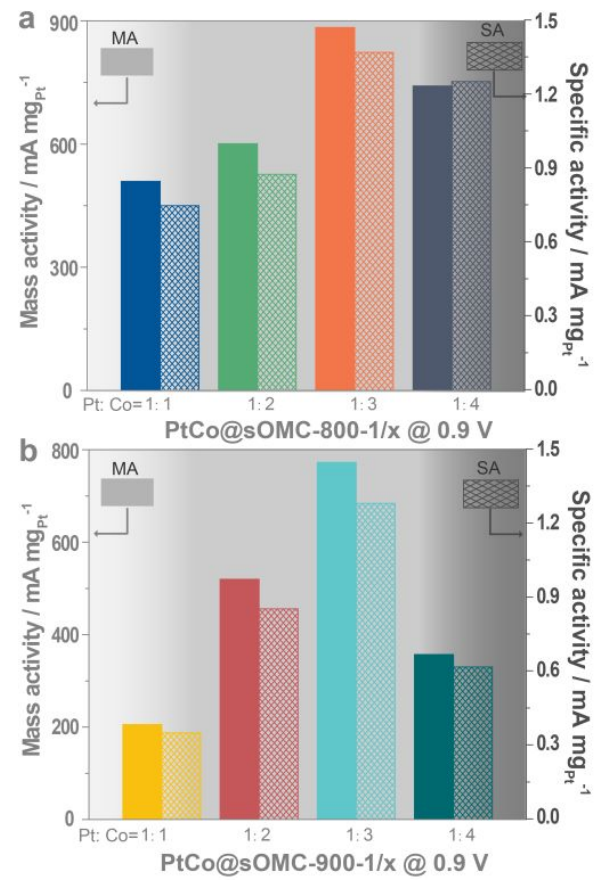

Figure S12. MA and SA at 0.9 V of a) PtCo@sOMC-800-1/x and b) PtCo@sOMC$900-1 / x$ catalysts 

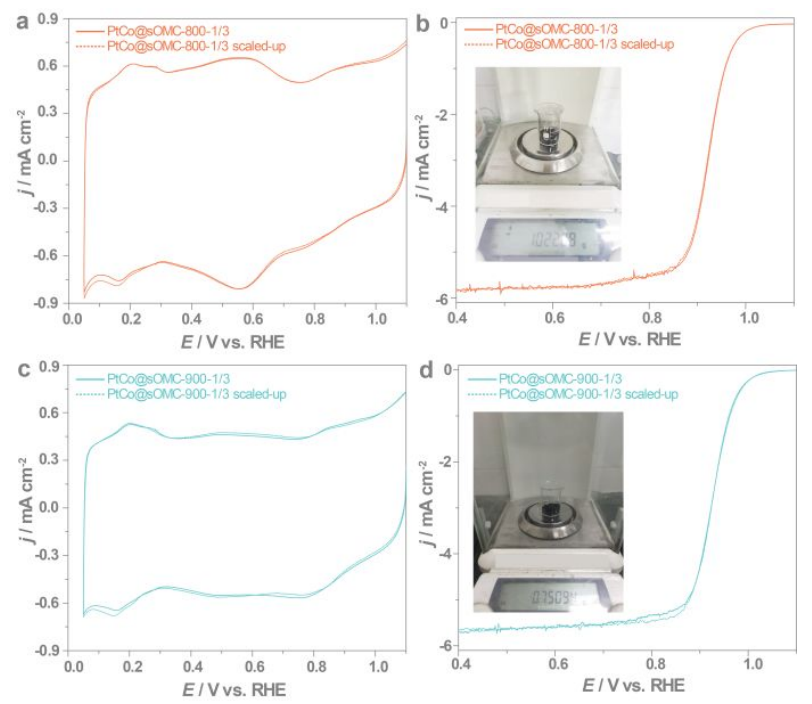

Figure S13. Catalytic performances of mass-produced PtCo@sOMC-800-1/3 and PtCo@sOMC-900-1/3 catalysts.
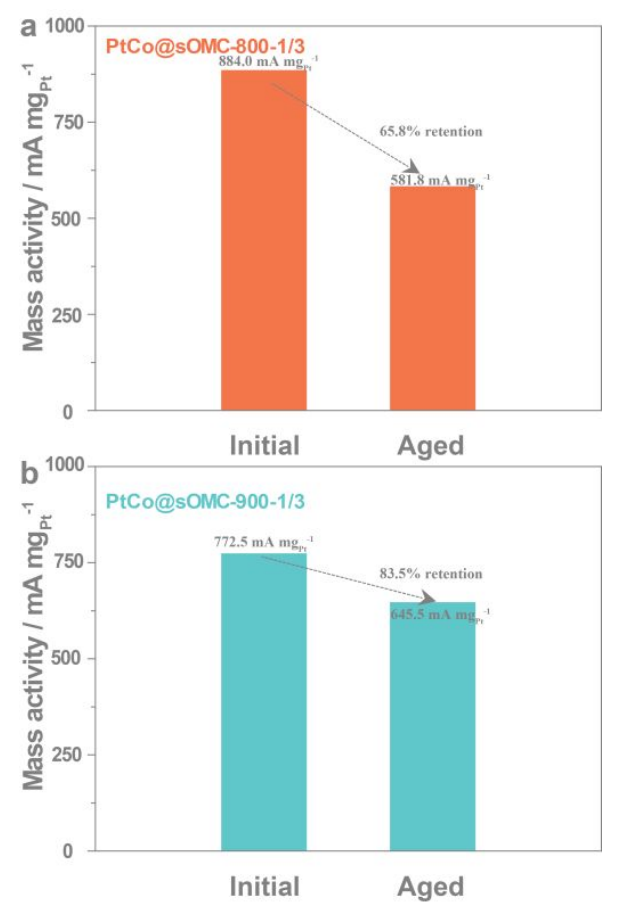

Figure S14. Mass activities at 0.9 V of a) PtCo@sOMC-800-1/3 and b) PtCo@sOMC-900-1/3 catalysts before and after ADTs.

Table S1. Catalytic performances of PtCo@sOMC-T-1/x catalysts.

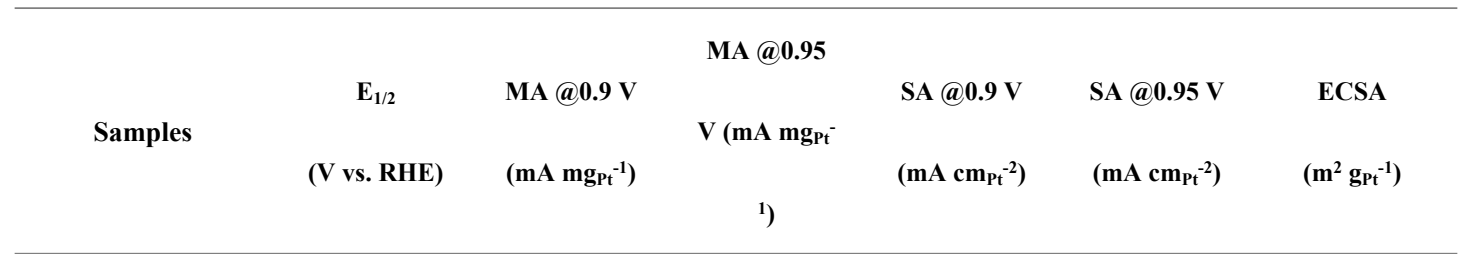




\begin{tabular}{ccccccc}
\hline PtCo@sOMC-300-1/2 & 0.857 & 84.1 & 12.2 & 0.116 & 0.017 & 72.4 \\
PtCo@sOMC-500-1/2 & 0.889 & 212.2 & 24.1 & 0.318 & 0.036 & 66.7 \\
PtCo@sOMC-700-1/2 & 0.909 & 400.8 & 51.1 & 0.609 & 0.078 & 65.8 \\
PtCo@sOMC-800-1/2 & 0.915 & 601.1 & 54.2 & 0.874 & 0.079 & 68.8 \\
PtCo@sOMC-900-1/2 & 0.913 & 519.6 & 57.2 & 0.852 & 0.094 & 61.0 \\
PtCo@sOMC-1100-1/2 & 0.889 & 207.9 & 25.4 & 0.393 & 0.048 & 52.9 \\
PtCo@sOMC-800-1/1 & 0.911 & 509.2 & 48.0 & 0.747 & 0.070 & 68.2 \\
PtCo@sOMC-800-1/3 & 0.923 & 884.0 & 75.1 & 1.368 & 0.116 & 64.6 \\
PtCo@sOMC-800-1/4 & 0.922 & 741.3 & 74.2 & 1.250 & 0.125 & 59.3 \\
PtCo@sOMC-900-1/1 & 0.891 & 205.8 & 32.1 & 0.349 & 0.054 & 59.1 \\
\hline PtCo@sOMC-900-1/3 & 0.923 & 772.5 & 90.4 & 1.280 & 0.150 & 60.3 \\
PtCo@sOMC-900-1/4 & 0.905 & 357.0 & 39.7 & 0.616 & 0.068 & 58.0 \\
\hline Pt@sOMC & 0.876 & 167.0 & 29.4 & 0.237 & 0.042 & 70.5 \\
\hline & 0.863 & 82.1 & 15.0 & 0.123 & 0.022 & 66.8 \\
\hline
\end{tabular}

Table S2. The proportion of C-containing Functionalities in PtCo@sOMC-T-1/x catalysts.

\begin{tabular}{|c|c|c|c|c|}
\hline Samples & C-sp2(\%) & C-sp3 (\%) & $\mathrm{C}-\mathrm{O}(\%)$ & $\mathrm{O}=\mathrm{C}-\mathrm{O}(\%)$ \\
\hline PtCo@sOMC-300-1/2 & 6.8 & 75.8 & 13.7 & 4.7 \\
\hline PtCo@sOMC-500-1/2 & 8.1 & 74.9 & 12.8 & 4.2 \\
\hline PtCo@sOMC-700-1/2 & 8.4 & 75.1 & 11.9 & 4.6 \\
\hline PtCo@sOMC-800-1/2 & 8.6 & 79.5 & 9.4 & 2.5 \\
\hline PtCo@sOMC-900-1/2 & 10.8 & 77.2 & 9.7 & 2.3 \\
\hline PtCo@sOMC-1100-1/2 & 16.4 & 73.4 & 8.0 & 2.2 \\
\hline PtCo@sOMC-800-1/1 & 6.9 & 70.4 & 14.9 & 7.8 \\
\hline PtCo@sOMC-800-1/3 & 9.1 & 79.1 & 9.3 & 2.5 \\
\hline PtCo@sOMC-800-1/4 & 9.2 & 81.6 & 6.9 & 2.3 \\
\hline PtCo@sOMC-900-1/1 & 9.2 & 78.8 & 8.8 & 3.2 \\
\hline PtCo@sOMC-900-1/3 & 11.5 & 78.5 & 7.9 & 2.1 \\
\hline PtCo@sOMC-900-1/4 & 17.9 & 71.5 & 8.3 & 2.3 \\
\hline
\end{tabular}


